\title{
Investigation of the geometric change of plastic articles made by polyamide
}

\author{
Metin Aclan ${ }^{1, *}$, Rauf Oezden ${ }^{1}$ and Saliba Danho $^{2}$ \\ ${ }^{1}$ Technical University of Cluj-Napoca, Department for Manufacturing Technology, Romania \\ 2 Johannes Gutenberg University Mainz, Germany
}

\begin{abstract}
The following paper includes an investigation of the geometric variation of plastic workpieces in a simulated environment of normal climate by $21 \circ / 50 \%$ relative humidity. In the industrial manufacturing, the daily quality checks are required to determine, if the plastic workpieces can be released. Due to the humidity or water absorption, the plastic workpiece grows so far that the dimensional accuracy is not given. In this investigation, the results of 12 weeks are determined and presented.
\end{abstract}

\section{Introduction}

In industrial production, it is necessary to carry out daily quality checks to determine whether plastic workpieces can be released.

In this case, the drawings which are produced according to standards or works standards, are valid as a reference. Thus, the measuring technology engineer, quality engineer or the machine operator is given fixed nominal dimensions and tolerances. In order to officially approve production, the plastic workpieces are measured again in the certified measuring room. Often, deviations from the previous measurements are detected.

Due to the absorption of moisture or water, the plastic workpiece expend in a way in which the dimensional accuracy can no longer be controlled. This often leads to unnecessary parameter changes on the tool, which the tool maintenance department has to carry out. In addition, design changes to the tool are always associated with very high costs.

The parts made of polyamide (PA66) have a higher hygroscopic expansion. Due to the many geometric differences of the plastic parts, there are no significant figures, data, and facts about the hygroscopic expansion effect on the dimensional accuracy of the respective part. In addition, the plastic part dimensional expansion prevents the reproducibility of measurements, making it impossible to make important decisions based on a fixed and reliable measurement.

In a globally active company with several production plants, these tests are carried out in the same way. Due to the different climatic conditions in the locations, different measured values are recorded. During the transports, which take one or two days, the plastic parts grow so much that the reproducibility of the measured values is prevented.

*Corresponding author: metin.aclan@googlemail.com 
In order to obtain a reproducibility of the measured values and thus to be able to make reliable quality decisions, it is necessary to know how much a plastic part can hygroscopic ally expand at all and which properties influence it.

\section{Causes of geometrical changes in plastics}

Plastics are an essential raw material of the numerous consumer products. In many areas and departments, competent experts deal with the problems of plastic material parts dimensional and geometrical instability. According to the manufacturers, computer software tries to simulate the occurring expansion as realistically as possible. Despite almost accurate results, the water absorption must be taken into account with regard to geometric modification. There are many factors that can affect the hygroscopic behavior of plastics. That is why it is important to understand the hygroscopic nature of plastic materials so that their behavior in the intended application can be anticipated. In order to get an overview of the causes of the geometric changes, it is important to understand which factors have the greatest influence on plastics. In addition, environmental influences such as temperature, humidity, as well as production-related factors [1] during injection molding, e.g:

- pressure,

- speed,

- cooling temperature,

- cooling time after the injection process.

These factors mainly influence the shrinkage that occurs after the injection of the plastic parts. In addition, the geometry is an important parameter that influences the part expansion levels. This means that greater growth can be prevented by reducing several free-form surfaces.

The knowledge is compared with the help of [2] whether the period of testing the plastic parts is well chosen. Thus, this report serves to deepen the theory in order to gain a more precise understanding of the interrelationships of the influences. Finally, the following is an outlook on the further experimental investigation of the various plastic articles.

\section{Tests and evaluations of different test criteria}

The basic conditions for the plastic workpieces are the following:

- Injection molding machines with hot runner;

- freshly molded and

- Series measurement max. 8 weeks.

In addition, the series measurements are to be carried out under various climatic conditions:

- $\quad$ climatic chamber $40^{\circ} \mathrm{C} / 93 \%$ rel. humidity;

- desiccator and

- other rooms for reference determination (room climate in the office, airconditioned room with standard climate $21^{\circ} \mathrm{C} / 50 \%$ rel. humidity).

\subsection{Determination of water absorption according to ISO 62}

After completion of the series measurements, the percentage water absorption defined by the weight of the respective materials in the various environments [3]. This is followed by an analysis of the plastic workpieces made of PA 66/6, PA 66/6 30\% GF, PBT. First, the individual environments are examined. Finally, an analysis of the materials behavior was 
carried out. The water intake determined from the series measurement for the selected materials are listed in Table 1 for an overview of the upcoming analysis.

Table 1. Determined water absorption after 8 weeks in the respective test environment.

\begin{tabular}{|c|c|c|c|c|c|}
\hline \multicolumn{6}{|c|}{ Moisture absorption in $\%$ after 8 weeks } \\
\hline \multicolumn{2}{|c|}{ Material PA 66/6 } & \multicolumn{4}{|c|}{ Test environment } \\
\hline \multirow[t]{2}{*}{ Distance in $\mathrm{mm}$} & \multirow[t]{2}{*}{79,05} & $\begin{array}{c}21^{\circ} \mathrm{C} 50 \% \\
\text { rel. } \\
\text { Humidity }\end{array}$ & $\begin{array}{c}40^{\circ} \mathrm{C} 93 \% \\
\text { rel. } \\
\text { Humidity }\end{array}$ & Desiccator & Indoor climate \\
\hline & & 2,39 & 8,2 & 0,47 & 2,05 \\
\hline \multicolumn{2}{|c|}{ Material PA $66 / 630 \%$ GF } & \multicolumn{4}{|c|}{ Test environment } \\
\hline \multirow[t]{2}{*}{ Distance in $\mathrm{mm}$} & \multirow[t]{2}{*}{63,24} & $\begin{array}{c}21^{\circ} \mathrm{C} 50 \% \\
\text { rel. } \\
\text { Humidity }\end{array}$ & $\begin{array}{c}40^{\circ} \mathrm{C} 93 \% \\
\text { rel. } \\
\text { Humidity }\end{array}$ & Desiccator & Indoor climate \\
\hline & & 1,44 & 3,39 & 0,31 & 1,34 \\
\hline \multicolumn{2}{|c|}{ Material PBT } & \multicolumn{4}{|c|}{ Test environment } \\
\hline \multirow[t]{2}{*}{ Distance in $\mathrm{mm}$} & \multirow[t]{2}{*}{107,25} & $\begin{array}{c}21^{\circ} \mathrm{C} 50 \% \\
\text { rel. } \\
\text { Humidity }\end{array}$ & $\begin{array}{c}40^{\circ} \mathrm{C} 93 \% \\
\text { rel. } \\
\text { Humidity }\end{array}$ & Desiccator & Indoor climate \\
\hline & & 0,18 & 0,41 & 0,05 & 0,17 \\
\hline
\end{tabular}

\subsubsection{Analysis of the test environment: $21^{\circ} \mathrm{C}, 50 \%$ rel. humidity (air-conditioned room):}

The values of the plastic workpieces with the material PA 66/6 correspond approximately to the manufacturer's guideline values of 2.6 to $3.2 \%$ and were to be expected accordingly.

The material polyamide $30 \%$ glass fiber reinforcement is $1.44 \%$ for both workpieces. This is clearly less than the workpieces of the standard polyamide $66 / 6$ without glass fiber reinforcement. The material PBT has the property of having low moisture absorption. The manufacturer gives a guideline value of $0.3 \%$ for this. After eight weeks of testing, the material has a moisture absorption of $0.18 \%$ and approximates the parameter of the manufacturer's guide value.

\subsubsection{Test environment analysis: $40^{\circ} \mathrm{C}, 93 \%$ rel. humidity:}

In this environment, all plastic workpieces reached saturation, i.e. maximum moisture absorption. The material PA $66 / 6$ has absorbed the most moisture (8 to 9\%). It is striking that the plastic part made of PA 66/6 has absorbed about two percent less moisture.

As in the previous environment, the material made of PA $66 / 630 \%$ of GF, in some cases even more than half the moisture content. The material that absorbed the least moisture was, as expected, the material made of PBT.

\subsubsection{Test environment analysis: Desiccator (for drying solid chemical substances):}

In the surroundings of the desiccator, it is evident that moisture absorption can be kept low for all plastic workpieces. Of all tested materials, PA 66/6 plastic workpieces have absorbed 
the most moisture with 0.38 to $0.50 \%$. This was followed by PA66/6 GF 30 with $0.31 \%$. This also shows lower moisture absorption than the PA 66/6. With 0.05 the material PBT of 0.02 , it again proves that moisture absorption can be kept to a minimum in the desiccator.

\subsubsection{Analysis of the test environment: indoor climate:}

The room climate as expected is similar to the test environment of the air-conditioned room.

\subsubsection{General Overview:}

The result of this evaluation shows the compressed knowledge about the moisture absorption of polyamides. The values of the material PA66/6 GF30 show that compared to the material of the unreinforced polyamide 66/6, they have absorbed considerably less (up to half of the moisture). The reason for this lies in the $30 \%$ glass fibers, which, among other things, ensure a higher density.

The degree of crystallization is also important [4]. Due to the cooling rate during the production of the workpieces, the degree of crystallization during processing can be varied up to $40 \%$.

This means that moisture and water absorption can be controlled through the parameter values, because the higher the degree of crystallization (higher density) of the plastic workpieces, the less water they absorb.

With a low humidity of 25 to $30 \%$ on average, which was present in the desiccator, the molding parts absorbed only minimal moisture as expected. Over the entire eight-week test period, the moisture absorption of the materials in the desiccator has therefore remained relatively low compared to the other environments.

The initial daily opening of the desiccator caused the humidity to rise between 35 to $40 \%$. In these phases, the plastic workpieces have absorbed most of their moisture. The moisture absorption in the environment of the room climate can be compared with that of the air-conditioned room at $21{ }^{\circ} \mathrm{C}, 50 \%$ rel. humidity. In the room climate, however, we have to mention that the temperature as well as the relative humidity varied during the test 19.5 to $22.5^{\circ} \mathrm{C}$ and 30.1 to $49.0 \%$.

From, this explains the lower moisture absorption, as the humidity was often lower than in the air-conditioned room. In addition, it must be noted and mentioned that the lower humidity is caused by the climatic conditions of the season (winter/spring), because then the humidity is usually $50 \%$ below that of the normal climate.

\subsection{Measurements of the selected linear dimensions}

In order to obtain an overview of all the data after the series measurements were carried out, they are displayed graphically with the aid of diagrams. Since the plastic material PA $66 / 6$ has been found to have the highest hygroscopic expansion, it was analyzed in detail.

The next figure shows the geometric changes during of the series measurement for the material PA 66/6. First of all, the overview is followed by a graphic representation of the plastic workpiece.

This includes the daily maximum expansion of the plastic workpiece in the various climatic environments listed in the legend of the graphics. In addition, the nominal size is indicated and included in the diagram. This is followed by an analysis of the plastic workpiece. 


\subsubsection{Material PA 66/6:}

The next figure 1 shows the course of the geometric change over a test period of eight weeks (nominal size $79.05 \mathrm{~mm} \pm 0.2 \mathrm{~mm}$ ).

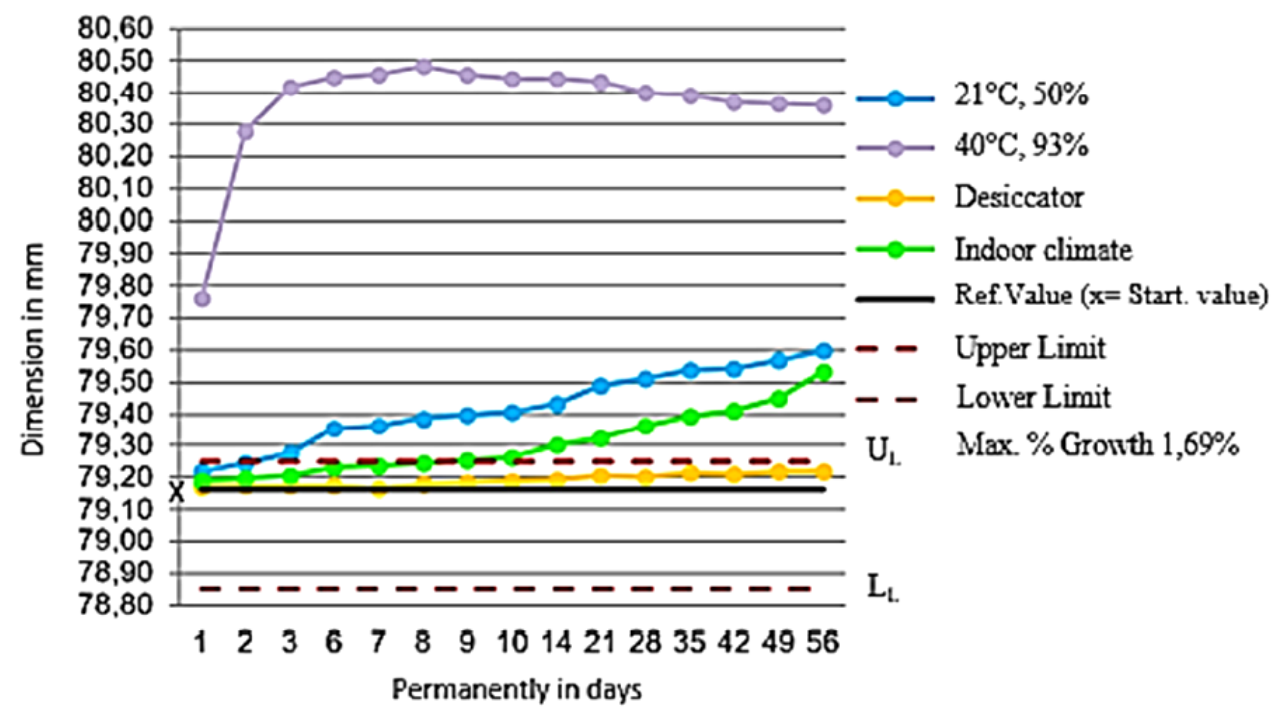

Fig. 1. Graphic representation of the geometric change PA66/6.

The reference value for the plastic article is $79.15 \mathrm{~mm}$. This value also results as average value of all reference values of the workpieces, as explained above. Looking at the graphic, it is immediately noticeable that in the environment with $40^{\circ} \mathrm{C}, 93 \%$ rel. humidity a growth of $0.61 \mathrm{~mm}$ has already occurred after one day and exceeds the upper tolerance limit. After approx. one week, the workpiece reaches its saturation at $80.49 \mathrm{~mm}$, corresponding to an expansion of $1.34 \mathrm{~mm}$.

Furthermore, a similar expansion in the environment of the air-conditioned room and the room climate can be seen. It is 0.45 and $0.38 \mathrm{~mm}$ for both. In this case, the upper tolerance limit for the air-conditioned room is exceeded after approx. two to three days and for the room climate after 10 days. There are no major modifications to the environment of the desiccator.

The growth in this environment is $0.07 \mathrm{~mm}$. Of all plastic workpieces, this one has the highest expansion the largest. This was expected in connection with the moisture absorption.

\section{Conclusions}

The evaluation of the test criteria has led to additional findings that demonstrate the hygroscopic expansion of the individual plastic materials in different environment specific conditions. Nevertheless, it is not possible to generalize the determined geometric instabilities for the plastic materials, but tends to be an accurate assumption, since expansion is still determined by the geometry of the component.

The most important new finding is that the acceptance according to [2] should take place after $16 \mathrm{~h}$ to $24 \mathrm{~h}$ at the earliest. However, this can only be achieved with the initial sampling, since measurements have to be taken within a short period of time (within the first hour/one to two hours) in production and in the quality departments. 
For this suggestion, a team of plastic experts, could design an instruction. In this context, it would be necessary to describe exactly how the plastics are to be handled. In the test report, replacement dimensions could be specified in the production (for immediate testing for the test dimensions) in this respect.

In addition, the tolerances should be adjusted during the immediate test, taking geometric instability into account.

Furthermore, it is shown that the free available tolerance window in which the operator for the injection molding machine can produce is decisive for the future dimensions. Additionally, the free available window determines, if or not the measurement is out of the tolerances at an early stage. Fixed parameters could be used to achieve standard dimensions which would result in better reproducibility of the values.

\section{References}

1. W. Kaiser, Plastics chemistry for engineers : from synthesis to the application, (2007) (in German)

2. DIN 16742, Plastics molded parts - Tolerances and acceptance conditions, (2013) (in German)

3. DIN 62, Plastics - Determination of water absorption, (2008) (in German)

4. S. Erguen, Dynamic oscillation behavior of amorphous bodies, (2015) (in German) 